\title{
CAPSULE COMMENTARIES \\ Capsule Commentary on Boissy et al., Communication Skills Training for Physicians Improves Patient Experiences
}

\author{
Sarah J. Nickoloff, MD \\ Zablocki VAMC, Milwaukee, WI, USA \\ $\mathrm{J}$ Gen Intern Med 31(7):778 \\ DOI: $10.1007 / \mathrm{s} 11606-016-3661-y$ \\ (c) Society of General Internal Medicine 2016
}

I $\mathrm{t}$ is well established that physician communication skills have a significant impact on both patient and provider satisfaction, ${ }^{1}$ and there is growing evidence that the level of satisfaction may affect clinical outcomes as well. ${ }^{2,3}$ The Centers for Medicare and Medicaid Services now require public reporting of patient experience scores through the Hospital Consumer Assessment of Healthcare Providers and Systems (HCAHPS) and the Clinician and Group Consumer Assessment of Healthcare Providers and Systems (CGCAHPS). ${ }^{4}$ These scores help determine reimbursement, and therefore have incentivized improvement in provider communication.

This observational study by Boissy and colleagues evaluated the impact on patient experience and physician satisfaction of a training course in experiential relationship-centered physician communications skills. ${ }^{5}$ Attending physicians at a large multispecialty academic medical center attended an 8-hour session that included interactive didactics, skill demonstrations, and small and large group skills practice sessions using the R.E.D.E. to Communicate: Foundations of Healthcare Communication training protocol. Physicians who had not taken the course or who had participated in an earlier version of the training served as the controls. The intervention group physicians showed higher adjusted CGCAHPS and HCAHPS scores following their training. The physicians also reported significant improvement in empathy and burnout. Some of these improvements were sustained at the 3-month follow-up mark.

This study does have some limitations that should be considered. As it was an observational study, the authors were unable to rule out other causes for the improvement in scores. Additionally, some outcomes were non-anonymous and selfreported, which, although unlikely, may have led to social desirability bias. Finally, it is unclear whether this model of training will be applicable across other settings that may employ fewer physicians or that are not affiliated with an academic institution.

Skilled physician communication is an important component of patient experience and provider satisfaction. This study presents a training model that improves patient experience scores, while at the same time increasing provider empathy and reducing fatigue. Given the small time investment for large benefit, it would be reasonable for systems that are seeking to improve these parameters to adopt a similar training model for their physicians.

Corresponding Author: Sarah J. Nickoloff, MD; Zablocki VAMC, Milwaukee, WI, USA (e-mail: Sarah.nickoloff@va.gov).

Compliance with Ethical Standards:

Conflict of Interest: The author has no conflicts of interest with this article.

\section{REFERENCES}

1. Jackson JL, Chamberlin J, Kroenke K. Predictors of Satisfaction. Soc Sci Med. 2001;52(4):609-20.

2. Stewart M, et al. the impact of patient-centered care on outcomes. J Fam Pract. 2000;49(9):796-804.

3. Roter DL, et al. Improving physicians' interviewing skills and reducing patients' emotional distress. A randomized clinical trial. Arch Intern Med. 1995;155(17): 1877-84

4. Hospital Consumer Assessment of Healthcare Providers and Systems. http://www.hcahpsonline.org. Accessed 2/3/2016.

5. Boissy A, et al. Communication skills training for physicians improves patient satisfaction. J Gen Intern Med. 2016. doi:10.1007/s11606-016-3597-2.

Published online March 10, 2016 